\title{
Chosen-Plaintext Cryptanalysis of a Clipped-Neural-Network-Based Chaotic Cipher ${ }^{\star}$
}

\author{
Chengqing $\mathrm{Li}^{1}$, Shujun $\mathrm{Li}^{2 a \star \star}$, Dan Zhang ${ }^{3}$ and Guanrong $\mathrm{Chen}^{2 b}$ \\ ${ }^{1}$ Department of Mathematics, Zhejiang University, Hangzhou 310027, China \\ 2 Department of Electronic Engineering, City University of Hong Kong, Kowloon, \\ Hong Kong, China \\ ${ }^{3}$ College of Computer Science, Zhejiang University, Hangzhou 310027, China
}

\begin{abstract}
In ISNN'04, a novel symmetric cipher was proposed, by combining a chaotic signal and a clipped neural network (CNN) for encryption. The present paper analyzes the security of this chaotic cipher against chosen-plaintext attacks, and points out that this cipher can be broken by a chosen-plaintext attack. Experimental analyses are given to support the feasibility of the proposed attack.
\end{abstract}

\section{Introduction}

Since the 1990s, the study of using chaotic systems to design new ciphers has become intensive [1]. In particular, the idea of combining chaos and neural networks has been developed [2], [3], [4], [5] and has been adopted for image and video encryption [6], [7]. In our recent work [8], it has been shown that the chaotic ciphers designed in [2], [3], [4], [6], [7] are not sufficiently secure from a cryptographical point of view.

This paper focuses on the security of a clipped-neural-network-based chaotic cipher proposed in ISNN'04 [5]. This chaotic cipher employs a chaotic pseudorandom signal and the output of a 8-cell clipped neural network to mask the plaintext, along with modulus additions and XOR operations. Also, the evolution of the neural network is controlled by the chaotic signal. With such a complicated combination, it was hoped that the chaotic cipher can resist chosenplaintext attacks. Unfortunately, our analysis shows that it is still not secure against chosen-plaintext attacks. By choosing only two plaintexts, an attacker can derive an equivalent key to break the cipher. This paper reports our analyses and simulation results.

The rest of the paper is organized as follows. Section 2 is a brief introduction to the chaotic cipher under study. The proposed chosen-plaintext attack is described in detail in Sec. 3, with some experimental results. The last section concludes the paper.

\footnotetext{
* This paper has been published in Advances in Neural Networks C ISNN 2005: Second International Symposium on Neural Networks, Chongqing, China, May 30 - June 1, 2005, Proceedings, Part II (ISNN 2005), Lecture Notes in Computer Science, vol. 3497, pp. 630-636.

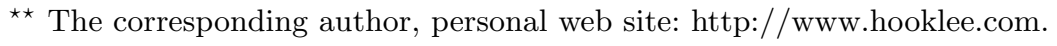




\section{The CNN-Based Chaotic Cipher}

First, the CNN employed in the chaotic cipher is introduced. The neural network contains 8 neural cells, denoted by $S_{0}, \cdots, S_{7} \in\{1,-1\}$, and each cell is connected with other cells via eight synaptic weights $w_{i j} \in\{1,0,-1\}$, among which only three are non-zeros. The synaptic weights between two connected cells are identical: $\forall i, j=0 \sim 7, w_{i j}=w_{j i}$. The neural network evolves according to the following rule: $\forall i=0 \sim 7$,

$$
f\left(S_{i}\right)=\operatorname{sign}\left(\widetilde{S}_{i}\right)= \begin{cases}1, & \widetilde{S}_{i}>0, \\ -1, & \widetilde{S}_{i}<0,\end{cases}
$$

where $\widetilde{S}_{i}=\sum_{j=0}^{7} w_{i j} S_{j}$. Note that $\widetilde{S}_{i} \neq 0$ holds at all times.

Now, let us see how the chaotic cipher works with the above CNN. Without loss of generality, assume that $f=\{f(i)\}_{i=0}^{N-1}$ is the plaintext signal, where $f(i)$ denotes the $i$-th plain-byte and $N$ is the plaintext size in byte. Accordingly, denote the ciphertext by $f^{\prime}=\left\{f^{\prime}(i)\right\}_{i=0}^{N-1}$, where $f^{\prime}(i)$ is a double-precision floating-point number corresponding to the plain-byte $f(i)$. The encryption procedure can be briefly depicted as follows ${ }^{1}$.

- The secret key includes the initial states of the 8 neural cells in the CNN, $S_{0}(0), \cdots, S_{7}(0)$, the initial condition $x(0)$, and the control parameter $r$ of the following chaotic tent map:

$$
T(x)= \begin{cases}r x, & 0<x \leq 0.5 \\ r(1-x), & 0.5<x<1\end{cases}
$$

where $r$ should be very close to 2 to ensure the chaoticity of the tent map.

- The initial procedure: 1) in double-precision floating-point arithmetic, run the tent map from $x(0)$ for 128 times before the encryption starts; 2) run the CNN for $128 / 8=16$ times (under the control of the tent map, as discussed below in the last step of the encryption procedure); 3) set $x(0)$ and $S_{0}(0), \cdots, S_{7}(0)$ to be the new states of the tent map and the CNN.

- The encryption procedure: for the $i$-th plain-byte $f(i)$, perform the following steps to get the ciphertext $f^{\prime}(i)$ :

- evolve the CNN for one step to get its new states: $S_{0}(i), \cdots, S_{7}(i)$;

- in double-precision floating-point arithmetic, run the chaotic tent map for 8 times to get 8 chaotic states: $x(8 i+0), \cdots, x(8 i+7)$;

- generate 8 bits by extracting the 4 -th bits of the 8 chaotic states: $b(8 i+$ $0), \cdots, b(8 i+7)$, and then $\forall j=0 \sim 7$, set $E_{j}=2 \cdot b(8 i+j)-1$;

- encrypt $f(i)$ as follows ${ }^{2}$ :

$$
f^{\prime}(i)=\left(\left(\frac{f(i) \oplus B(i)}{256}+x(8 i+7)\right) \bmod 1\right),
$$

\footnotetext{
${ }^{1}$ Note that some original notations used in [5] have been changed in order to provide a better description.

${ }^{2}$ In $[5], x(8 i+7)$ was mistaken as $x(8)$.
} 
where $B(i)=\sum_{j=0}^{7}\left(\frac{S_{j}(i)+1}{2}\right) \cdot 2^{7-j}$

- $\forall i=0 \sim 7$, if $S_{i} \neq E_{i}$, update all the three non-zero weights of the $i$-th neural cell and the three mirror weights as follows: $w_{i j}=-w_{i j}$, $w_{j i}=-w_{j i}$.

- The decryption procedure is similar to the above one with the following decryption formula:

$$
f(i)=\left(256 \cdot\left(\left(f^{\prime}(i)-x(8 i+7)\right) \bmod 1\right)\right) \oplus B(i) .
$$

\section{The Chosen-Plaintext Attack}

In chosen-plaintext attacks, it is assumed that the attacker can intentionally choose a number of plaintexts to try to break the secret key or its equivalent [9]. Although it was claimed that the chaotic cipher under study can resist this kind of attacks [5, Sec. 4], our cryptanalysis shows that such a claim is not true. By choosing two plaintexts, $f_{1}$ and $f_{2}$, satisfying $\forall i=0 \sim N-1, f_{1}(i)=\overline{f_{2}(i)}$, one can derive two masking sequences as equivalent keys for decryption.

Before introducing the chosen-plaintext attack, three lemmas are given, which are useful in the following discussions.

Lemma 1. $\forall a, b, c \in \mathbb{R}, c \neq 0$ and $n \in \mathbb{Z}^{+}$, if $a=(b \bmod c)$, one has $a \cdot n=$ $((b \cdot n) \bmod (c \cdot n))$.

Proof. From $a=(b \bmod c)$, one knows that $\exists k \in \mathbb{Z}, b=c \cdot k+a$ and $0 \leq a<c$. Thus, $\forall n \in \mathbb{Z}^{+}, b \cdot n=c \cdot n \cdot k+a \cdot n$ and $0 \leq a \cdot n<c \cdot n$, which immediately leads to $a \cdot n=((b \cdot n) \bmod (c \cdot n))$ and completes the proof of this lemma.

Lemma 2. $\forall a, b, c, n \in \mathbb{R}$ and $0 \leq a, b<n$, if $c=((a-b) \bmod n)$, one has $a-b \in\{c, c-n\}$.

Proof. This lemma can be proved under two conditions. i) When $a \geq b$, it is obvious that $((a-b) \bmod n)=a-b=c$. ii) When $a<b,((a-b) \bmod n)=$ $((n+a-b) \bmod n)$. Since $-n<a-b<0$, one has $0<n+a-b<n$, which means that $((a-b) \bmod n)=n+a-b=c$. That is, $a-b=c-n$. Combining the two conditions, this lemma is thus proved.

Lemma 3. Assume that $a, b$ are both 8-bit integers. If $a=b \oplus 128$, then $a \equiv$ $(b+128)(\bmod 256)$.

Proof. This lemma can be proved under two conditions. i) When $0 \leq a<128$ : $b=a \oplus 128=a+128$, so $a \equiv(b+128)(\bmod 256)$. ii) When $128 \leq a \leq 255$ : $b=a \oplus 128=a-128$, so $a \equiv(b-128) \equiv(b+128)(\bmod 256)$.

From Lemma 1, one can rewrite the encryption formula Eq. (3) as follows:

$$
256 \cdot f^{\prime}(i)=(((f(i) \oplus B(i))+256 \cdot x(8 i+7)) \bmod 256) .
$$


Given two plain-bytes $f_{1}(i) \neq f_{2}(i)$ and the corresponding cipher-blocks $f_{1}^{\prime}(i), f_{2}^{\prime}(i)$, one has $256 \cdot\left(f_{1}^{\prime}(i)-f_{2}^{\prime}(i)\right) \equiv\left(\left(f_{1}(i) \oplus B(i)\right)-\left(f_{2}(i) \oplus B(i)\right)\right) \quad(\bmod 256)$. Without loss of generality, assume that $f_{1}^{\prime}(i)>f_{2}^{\prime}(i)$ and that $\Delta_{f_{1,2}}=256 \cdot\left(f_{1}^{\prime}(i)-\right.$ $\left.f_{2}^{\prime}(i)\right)$. It is true that $0<\Delta_{f_{1,2}}<256$. Thus, one has

$$
\Delta_{f_{1,2}}=\left(\left(\left(f_{1}(i) \oplus B(i)\right)-\left(f_{2}(i) \oplus B(i)\right)\right) \bmod 256\right) .
$$

Because $f_{1}(i) \oplus B(i)$ and $f_{2}(i) \oplus B(i)$ are 8-bit integers and $\Delta_{f_{1,2}} \neq 0$, from Lemma 2 , one of the following facts is true:

$$
\begin{aligned}
& \text { 1. }\left(f_{1}(i) \oplus B(i)\right)-\left(f_{2}(i) \oplus B(i)\right)=\Delta_{f_{1,2}} \in\{1, \cdots, 255\} \\
& \text { 2. }\left(f_{2}(i) \oplus B(i)\right)-\left(f_{1}(i) \oplus B(i)\right)=\left(256-\Delta_{f_{1,2}}\right) \in\{1, \cdots, 255\}
\end{aligned}
$$

For the above two equations, when $f_{1}(i)=\overline{f_{2}(i)}$ is satisfied, two possible values of $B(i)$ can be uniquely derived according to the following theorem.

Theorem 1. Assume that $a, b, c, x$ are all 8-bit integers, and $c>0$. If $a=$ $\bar{b}$, then the equation $(a \oplus x)-(b \oplus x)=c$ has an unique solution $x=a \oplus$ $\left(1, c_{7}, \cdots, c_{1}\right)_{2}$, where $c=\left(c_{7}, \cdots, c_{0}\right)_{2}=\sum_{i=0}^{7} c_{i} \cdot 2^{i}$.

Proof. Since $a=\bar{b}$, one has $b \oplus x=\overline{a \oplus x}$. Thus, by substituting $y=a \oplus x$ and $\bar{y}=\overline{a \oplus x}=b \oplus x$ into $(a \oplus x)-(b \oplus x)=c$, one can get $y-\bar{y}=c$, which is equivalent to $y=\bar{y}+c$. Let $y=\sum_{i=0}^{7} y_{i} \cdot 2^{i}$, and consider the following three conditions, respectively.

1) When $i=0$, from $y_{0} \equiv\left(\bar{y}_{0}+c_{0}\right)(\bmod 2)$, one can immediately get $c_{0}=1$. Note the following two facts: i) when $y_{0}=0, \bar{y}_{0}+c_{0}=2$, a carry bit is generated for the next bit, so $y_{1} \equiv\left(\bar{y}_{1}+c_{1}+1\right)(\bmod 2)$ and $c_{1}=0$; ii) when $y_{0}=1$, $\overline{y_{0}}+c_{0}=1$, no carry bit is generated, so $y_{1} \equiv\left(\bar{y}_{1}+c_{1}\right)(\bmod 2)$ and $c_{1}=1$. Apparently, it is always true that $y_{0}=c_{1}$. Also, a carry bit is generated if $c_{1}=0$ is observed.

2) When $i=1$, if there exists a carry bit, set $c_{1}^{\prime}=c_{1}+1 \in\{1,2\}$; otherwise, set $c_{1}^{\prime}=c_{1} \in\{0,1\}$. From $y_{1} \equiv\left(\bar{y}_{1}+c_{1}^{\prime}\right)(\bmod 2)$, one can immediately get $c_{1}^{\prime}=1$. Then, using the same method shown in the first condition, one has $y_{1}=c_{2}$ and knows whether or not a carry bit is generated for $i=2$. Repeat the above procedure for $i=2 \sim 6$, one can uniquely determine that $y_{i}=c_{i+1}$.

3) When $i=7$, it is always true that the carry bit does not occur, so $c_{7}^{\prime}=1$, and $y_{7} \equiv 1$.

Combining the above three conditions, one can get $y=\left(1, c_{7}, \cdots, c_{1}\right)_{2}$, which results in $x=a \oplus\left(1, c_{7}, \cdots, c_{1}\right)_{2}$.

Assume that the two values of $B(i)$ derived from Eqs. (7a) and (7b) are $B_{1}(i)$ and $B_{2}(i)$, respectively. The following corollary shows that the two values have a deterministic relation: $B_{2}(i)=B_{1}(i) \oplus 128$.

Corollary 1. Assume that $a, b, c, x$ are all 8 -bit integers, $a=\bar{b}$ and $c>0$. Given two equations, $(a \oplus x)-(b \oplus x)=c$ and $\left(b \oplus x^{\prime}\right)-\left(a \oplus x^{\prime}\right)=c^{\prime}$, if $c^{\prime}=256-c$, then $x^{\prime}=x \oplus 128$. 
Proof. Since $c+\bar{c}=255$, one has $c^{\prime}=256-c=\bar{c}+1$. Let $c=\sum_{i=0}^{7} c_{i} \cdot 2^{i}$, and observe the first condition of the proof of Theorem 1. One can see that $c_{0}=1$, so $c_{0}^{\prime}=\bar{c}_{0}+1=1$. Since there is no carry bit, one can deduce that $\forall i=1 \sim 7$, $c_{i}^{\prime}=\bar{c}_{i}$. Applying Theorem 1 for $(a \oplus x)-(b \oplus x)=c$, one can uniquely get $x=a \oplus\left(1, c_{7}, \cdots, c_{1}\right)_{2}$. Then, applying Theorem 1 for $\left(b \oplus x^{\prime}\right)-\left(a \oplus x^{\prime}\right)=c^{\prime}$, one has $x^{\prime}=b \oplus\left(1, c_{7}^{\prime}, \cdots, c_{1}^{\prime}\right)_{2}=\bar{a} \oplus\left(1, \bar{c}_{7}, \cdots, \bar{c}_{1}\right)_{2}=\left(a_{7}, \bar{a}_{6} \oplus \bar{c}_{7}, \cdots, \bar{a}_{0} \oplus \bar{c}_{1}\right)_{2}=$ $\left(a_{7}, a_{6} \oplus c_{7}, \cdots, a_{0} \oplus c_{1}\right)_{2}=a \oplus\left(1, c_{7}, \cdots, c_{1}\right)_{2} \oplus(1,0, \cdots, 0)_{2}=x \oplus 128$. Thus, this corollary is proved.

For any one of the two candidate values of $B(i)$, one can further get an equivalent chaotic state $\hat{x}(8 i+7)$ from $B(i), f(i)$ and $f^{\prime}(i)$ as follows:

$$
\hat{x}(8 i+7)=256 \cdot f^{\prime}(i)-(f(i) \oplus B(i)) \equiv 256 \cdot x(8 i+7) \quad(\bmod 256) .
$$

With $B(i)$ and $\hat{x}(8 i+7)$, the encryption formula Eq. (3) becomes

$$
f^{\prime}(i)=\frac{((f(i) \oplus B(i))+\hat{x}(8 i+7)) \bmod 256}{256},
$$

and the decryption formula Eq. (4) becomes

$$
f(i)=\left(\left(256 \cdot f^{\prime}(i)-\hat{x}(8 i+7)\right) \bmod 256\right) \oplus B(i) .
$$

Assume that $\hat{x}_{1}(8 i+7)$ and $\hat{x}_{2}(8 i+7)$ are calculated by Eq. (8), from $B_{1}(i)$ and $B_{2}(i)$, respectively. Then, we have the following proposition.

Proposition 1. $\left(B_{1}(i), \hat{x}_{1}(8 i+7)\right)$ and $\left(B_{2}(i), \hat{x}_{2}(8 i+7)\right)$ are equivalent for the above encryption procedure Eq. (9), though only one corresponds to the correct value generated from the secret key. That is,

$$
\left(\left(f(i) \oplus B_{1}(i)\right)+\hat{x}_{1}(8 i+7)\right) \equiv\left(\left(f(i) \oplus B_{2}(i)\right)+\hat{x}_{2}(8 i+7)\right) \quad(\bmod 256) .
$$

Proof. From $B_{1}(i)=B_{2}(i) \oplus 128$, one has $f(i) \oplus B_{1}(i)=\left(f(i) \oplus B_{2}(i) \oplus 128\right)$. Then, following Lemma 3 , it is true that $\left(f(i) \oplus B_{1}(i)\right) \equiv\left(\left(f(i) \oplus B_{2}(i)\right)+\right.$ 128) $(\bmod 256)$. As a result, $\hat{x}_{1}(8 i+7)=\left(256 \cdot f^{\prime}(i)-\left(f(i) \oplus B_{1}(i)\right)\right) \equiv$ $\left(256 \cdot f^{\prime}(i)-\left(\left(f(i) \oplus B_{2}(i)\right)-128\right)\right)(\bmod 256) \equiv\left(\hat{x}_{2}(8 i+7)+128\right)(\bmod 256)$, which immediately leads to the following fact: $\left(\left(f(i) \oplus B_{1}(i)\right)+\hat{x}_{1}(8 i+7)\right) \equiv$ $\left(\left(f(i) \oplus B_{2}(i)\right)+\hat{x}_{2}(8 i+7)\right) \quad(\bmod 256)$. Thus, this proposition is proved.

Considering the symmetry of the encryption and decryption procedures, the above proposition immediately leads to a conclusion that $\left(B_{1}(i), \hat{x}_{1}(8 i+7)\right)$ and $\left(B_{2}(i), \hat{x}_{2}(8 i+7)\right)$ are also equivalent for the decryption procedure Eq. (10).

From the above analyses, with two chosen plaintexts $f_{1}$ and $f_{2}=\bar{f}_{1}$, one can get the following two sequences: $\left\{B_{1}(i), \hat{x}_{1}(8 i+7)\right\}_{i=0}^{N-1}$ and $\left\{B_{2}(i), \hat{x}_{2}(8 i+\right.$ 7) $\}_{i=0}^{N-1}$. Given a ciphertext $f^{\prime}=\left\{f^{\prime}(i)\right\}_{i=0}^{N-1}, \forall i=0 \sim N-1$, one can use either $\left(B_{1}(i), \hat{x}_{1}(8 i+7)\right)$ or $\left(B_{2}(i), \hat{x}_{2}(8 i+7)\right)$ as an equivalent of the secret key to decrypt the $i$-th plain-byte $f(i)$, following Eq. (10). This means that the chaotic cipher under study is not sufficiently secure against the chosen-plaintext attack. 
To demonstrate the feasibility of the proposed attack, some experiments have been performed for image encryption, with secret key $r=1.99, x(0)=0.41$ and $\left[S_{0}(0), \cdots, S_{7}(0)\right]=[1,-1,1,-1,1,-1,1,-1]$. One plain-image "Lenna" of size $256 \times 256$ is chosen as $f_{1}$ and another plain-image is manually generated as follows: $f_{2}=\bar{f}_{1}$. The two plain-images and their cipher-images are shown in Fig. 1. With the two chosen plain-images, two sequences, $\left\{B_{1}(i), \hat{x}_{1}(8 i+\right.$ 7) $\}_{i=0}^{256 \times 256-1}$ and $\left\{B_{2}(i), \hat{x}_{2}(8 i+7)\right\}_{i=0}^{256 \times 256-1}$, are generated by using the abovementioned algorithm. The first ten elements of the two sequences are given in Table 1. $\forall i=0 \sim(256 \times 256-1)$, either $\left(B_{1}(i), \hat{x}_{1}(8 i+7)\right)$ or $\left(B_{2}(i), \hat{x}_{2}(8 i+7)\right)$ can be used to recover the plain-byte $f(i)$. As a result, the whole plain-image ("Peppers" in this test) can be recovered as shown in Fig. 1f.

Table 1. The first ten elements of $\left\{B_{1}(i), \hat{x}_{1}(8 i+7)\right\}_{i=0}^{256 \times 256-1}$ and $\left\{B_{2}(i), \hat{x}_{2}(8 i+\right.$ 7) $\}_{i=0}^{256 \times 256-1}$

\begin{tabular}{c|c|c|c|c|c|c|c|c|c|c}
\hline$i$ & 0 & 1 & 2 & 3 & 4 & 5 & 6 & 7 & 8 & 9 \\
\hline$B_{1}(i)$ & 146 & 231 & 54 & 202 & 59 & 243 & 166 & 173 & 233 & 82 \\
\hline$B_{2}(i)$ & 18 & 103 & 182 & 74 & 187 & 115 & 38 & 45 & 105 & 210 \\
\hline$\hat{x}_{1}(8 i+7)$ & 242.40 & 38.63 & 242.62 & 222.09 & 81.03 & 214.73 & 240.91 & 203.59 & 138.20 & 9.33 \\
\hline$\hat{x}_{2}(8 i+7)$ & 114.40 & 166.63 & 114.62 & 94.09 & 209.03 & 86.73 & 112.91 & 75.59 & 10.20 & 137.33 \\
\hline
\end{tabular}

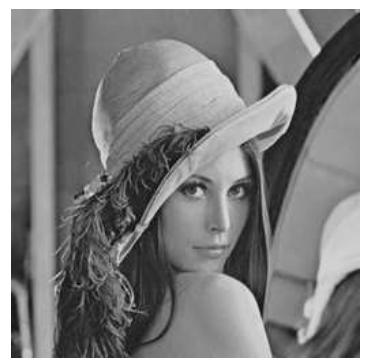

a) Chosen plain-image $f_{1}$

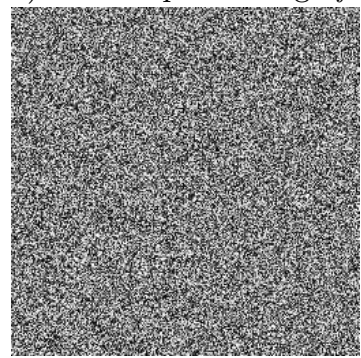

b) Cipher-image $f_{1}^{\prime}$

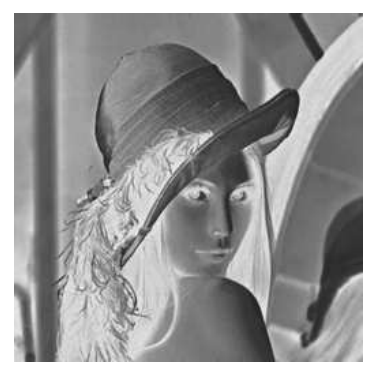

c) Chosen plain-image $f_{2}$

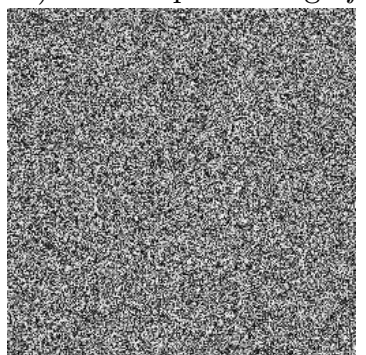

d) Cipher-image $f_{2}^{\prime}$

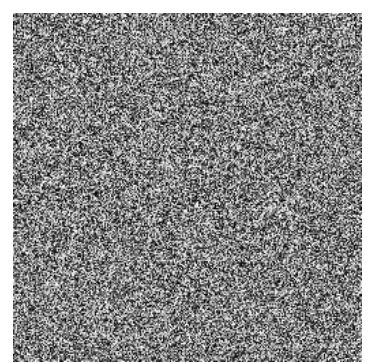

e) A cipher-image $f_{3}^{\prime}$

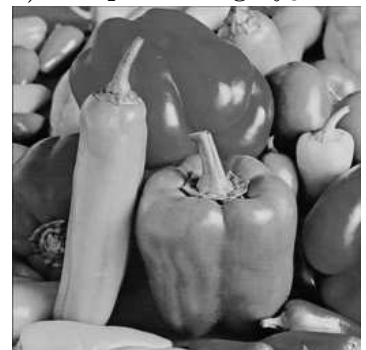

f) Recovered image $f_{3}$

Fig. 1. The proposed chosen-plaintext attack 


\section{Conclusion}

In this paper, the security of a chaotic cipher based on clipped neural network has been analyzed in detail. It is found that the scheme can be effectively broken with only two chosen plain-images. Both theoretical and experimental analyses have been given to support the proposed attack. Therefore, this scheme is not suggested for applications that requires a high level of security.

Acknowledgements. This research was supported by the National Natural Science Foundation, China, under grant no. 60202002, and by the Applied R\&D Centers of the City University of Hong Kong under grants no. 9410011 and no. 9620004 .

\section{References}

1. Li, S.: Analyses and New Designs of Digital Chaotic Ciphers. PhD thesis, School of Electronics and Information Engineering, Xi'an Jiaotong University, Xi'an, China (2003) available online at http://www.hooklee.com/pub.html.

2. Yen, J.C., Guo, J.I.: A chaotic neural network for signal encryption/decryption and its VLSI architecture. In: Proc. 10th VLSI Design/CAD Symposium. (1999) 319-322

3. Su, S., Lin, A., Yen, J.C.: Design and realization of a new chaotic neural encryption/decryption network. In: Proc. IEEE Asia-Pacific Conference on Circuits and Systems. (2000) 335-338

4. Yen, J.C., Guo, J.I.: The design and realization of a chaotic neural signal security system. Pattern Recognition and Image Analysis (Advances in Mathematical Theory and Applications) 12 (2002) 70-79

5. Zhou, T., Liao, X., Chen, Y.: A novel symmetric cryptography based on chaotic signal generator and a clipped neural network. In: Advances in Neural Networks - ISNN 2004: International Symposium on Neural Networks Proceedings, Part II. Volume 3174 of Lecture Notes in Computer Science. (2004) 639-644

6. Lian, S., Chen, G., Cheung, A., Wang, Z.: A chaotic-neural-network-based encryption algorithm for JPEG2000 encoded images. In: Advances in Neural Networks ISNN 2004 Proceedings, Part II. Volume 3174 of Lecture Notes in Computer Science. (2004) 627-632

7. Lian, S., Sun, J., Li, Z., Wang, Z.: A fast MPEG4 video encryption scheme based on chaotic neural network. In: Neural Information Processing: ICONIP 2004 Proceedings. Volume 3316 of Lecture Notes in Computer Science. (2004) 720-725

8. Li, C., Li, S., Zhang, D., Chen, G.: Cryptanalysis of a chaotic neural network based multimedia encryption scheme. In: Advances in Multimedia Information Processing - PCM 2004 Proceedings, Part III. Volume 3333 of Lecture Notes in Computer Science., Springer-Verlag (2004) 418-425

9. Schneier, B.: Applied Cryptography - Protocols, Algorithms, and Souce Code in C. Second edn. John Wiley \& Sons, Inc., New York (1996) 